\title{
Investing in Relationships and Teams to Support Managing Complexity
}

\author{
Shari D. Bolen, $M D, M P H^{1,2}$ and Kurt C. Stange, $M D, P h D^{3}$ \\ 'Department of Medicine, MetroHealth Medical Center, Cleveland, OH, USA; ${ }^{2}$ Center for Health Care Research and Policy, Case Western Reserve \\ University at MetroHealth Medical Center, Cleveland, OH, USA; ${ }^{3}$ Department of Family Medicine \& Community Health, Case Western Reserve \\ University, Cleveland, $\mathrm{OH}$, USA.
}

$\mathrm{J}$ Gen Intern Med 32(3):241-2

DOI: $10.1007 / \mathrm{s} 11606-016-3959-9$

(c) Society of General Internal Medicine 2016

$\mathrm{C}$ ontrary to some misperceptions, primary care is not about dealing with simple diseases. ${ }^{1}$ Primary care involves caring for individuals with multiple acute, chronic, preventive, mental health, and socioeconomic concerns. ${ }^{2}$ It is about developing the deep personal knowledge and relationships necessary to diagnose complex problems that don't fit into neat little boxes, and to help people and the system to manage complexity. ${ }^{3}$ The ability to manage complexity helps explain the paradox of primary care-the observation that primary care physicians provide poorer quality of care for specific diseases than do specialists, yet primary care is associated with better health, greater equity, lower costs, and better quality of care at the whole-person and population level. ${ }^{3}$ The current fragmented health care system desperately needs to support primary care to manage patient complexity, if it is to improve the health of the whole person and the health of populations and subpopulations. ${ }^{4}$

In this issue of JGIM, Hwang and colleagues ${ }^{5}$ describe and compare four patient types from the primary care provider perspective: 1) high-effort, 2) high-complexity, 3) high-effort and high-complexity, and 4) loweffort and low-complexity. They report that primary care providers at one large academic hospital center in Boston classified a large percentage of their patients as high-effort (24\%) and/or complex (28\%), with one in five patients being both high-effort and highly complex. In contrast, many patients $(68 \%)$ were seen as low effort or not complex. The authors also evaluate patient characteristics associated with these four categories of patients, reporting that high-effort patients have higher rates of medical and psychosocial health problems and more health care utilization. When distinguishing high effort only versus high complexity only, Hwang and colleagues note that high-effort patients were more likely to exhibit substance use and use more outpatient

Published online December 21, 2016 visits (both to primary care and non-primary care providers). The findings show that high effort and higher complexity are not always the same thing.

Managing complex or high-effort patients requires the integration of different perspectives to facilitate co-learning among patients, clinicians, and the health care team. ${ }^{6}$ The ability to manage complexity is enhanced by investment on the part of patients, practices, and systems in developing relationships, even during encounters that are not complex, so that a "relationship well" can be drawn upon to integrate, personalize, and prioritize care when things become complex. However, current simplistic, often disease-specific ways of measuring quality of care are blind to the benefits of this investment in relationship, and may unintentionally devalue connection in favor of "productivity" crudely measured as the number of patients seen per unit of time.,

In addition to a personal relationship, managing complexity benefits from linkages to deep and diverse resources. The health care team may notice improved outcomes among a subgroup of their patients and promote incorporation of interventions or collaborations with community resources from this learning to improve the care of their other patients.

Interventions aimed at redesigning primary care to address the needs of complex or high-effort patients have increased in the last decade in response to concerns about reducing the cost of care. Several of these interventions have focused on what are often called "superutilizers," or those patients with frequent hospitalizations and emergency room visits. Other interventions have focused on patients with multi-morbidity. A recent Cochrane review ${ }^{9}$ of interventions for patients with multi-morbid conditions suggests that enhanced care teams addressing care coordination, individualized care plans, and patient self-management for specific diseases may have the greatest effect, while patient selfmanagement interventions separate from the health care system or broad health care system redesign without focused quality improvement targets are less effective. In addition, interventions clearly need to incorporate expertise in addiction, mental health, and social needs, given the increased prevalence of these issues in higheffort or complex populations. Primary care teams (including patients) should be at the forefront of these 
health care system redesign conversations, since they are best prepared to contextualize the redesign to best suit their clinic team within the context of their community.

Over half of U.S. physicians reported at least one symptom of burnout in 2014, with frontline physicians such as primary care physicians having one of the highest burnout rates. Addressing complex or high-effort patients is challenging, and changing health care systems alone requires increased effort on the part of a primary care team. During health care redesign, health care system leaders need to take a preventive approach to burnout by investing in practices' adaptive reserve. ${ }^{10}$ Efforts to compel primary care teams to focus only on the most challenging, complex, or high-effort patients are likely to further burnout. ${ }^{11}$ However, adequately supported team approaches to care, and supporting the time during simpler visits to help develop the understanding and relationships that can be drawn on to help primary care clinicians manage subsequent complexity, might reduce burnout and is a worthy investment for the health care system.

In addition to describing complex or high-effort patients, Hwang and colleagues discuss how better risk adjustment for complex or high-effort patients could be used for enhanced reimbursement strategies. Enhanced reimbursement for the additional care coordination and team-based care required by these patients compared to lower-effort or less complex patients is critical for improving patient care and reducing burnout. The study by Hwang and colleagues supports increasing efforts to recognize ${ }^{12,13}$ and develop mechanisms to support management of multimorbidity, ${ }^{9}$ which is the rule rather than the exception in primary care. ${ }^{14}$

This study leads to several important yet unanswered questions which require future investigation. First, how stable is a patient's residence in one of the four categories formed by crossing complexity with effort? Second, do certain primary care approaches (particularly ones that are largely invisible to current quality and "productivity" measures, such as investing in the relationship) affect progression to greater complexity? Third, how do the primary care tenets (accessibility, wholeperson focus, coordination and integration of care, partnership) buffer the patient from the adverse effects of a fragmented health care system, and buffer the system from high and inappropriate use of singly focused (and sometimes risky) diagnostic and therapeutic interventions for complexly related problems?

In summary, understanding, managing, and helping others to manage complex or high-effort patients is a quintessential feature of primary care, and an under-recognized source of its value to individuals, families, and communities, as well as to system effectiveness and sustainability. ${ }^{15}$ The current overly simplistic measurement culture devalues primary care's sophisticated role in managing complex or high-effort patients. Measures and support mechanisms need to recognize this complexity, and reward, rather than punish, those who are willing to deal with it. ${ }^{16}$ Efforts to support bringing the social determinants of health into health care can help primary care to manage complexity, if these efforts are appropriately resourced. Managing complexity involves investing in the more basic, seemingly simpler levels of care, where providers develop deep understanding and relationships with patients that can be drawn upon for the higher-level primary care functions of integrating, personalizing, and prioritizing care, and abiding with people to advance healing. ${ }^{17}$

Acknowledgements: Dr. Stange is financially supported as a Scholar of The Institute for Integrative Health and as a Clinical Research Professor for the American Cancer Society.

Corresponding Author: Shari D. Bolen, MD, MPH; Center for Health Care Research and PolicyCase Western Reserve University at MetroHealth Medical Center, 2500 MetroHealth Drive, Rammelkamp Building, room R234A, Cleveland, OH 44109, USA (e-mail: sdb73@case.edu).

\section{Compliance with Ethical Standards:}

Conflict of Interest: The authors declare that they do not have a conflict of interest.

\section{REFERENCES}

1. Katerndahl DA, Wood R, Jaen CR. A method for estimating relative complexity of ambulatory care. Ann Fam Med. 2010;8(4):341-7.

2. Bolen SD, Sage P, Perzynski AT, Stange KC. No moment wasted: the primary-care visit for adults with diabetes and low socio-economic status. Prim Health Care Res Dev. 2016;17(1):18-32.

3. Stange KC, Ferrer RL. The paradox of primary care. Ann Fam Med. 2009;7(4):293-9.

4. Stange $\mathbf{K C}$. The problem of fragmentation and the need for integrative solutions. Ann Fam Med. 2009;7(2):100-3.

5. Hwang ASAS, Hong $\mathbf{J}$, et al. Defining team effort involved in patient care from the primary care physician's perspective. J Gen Intern Med. 2016. doi:10.1007/s11606-016-3897-6.

6. Stange KC. Ways of knowing, learning, and developing. Ann Fam Med. 2010;8(1):4-10.

7. Werner RM, Greenfield S, Fung C, Turner BJ. Measuring quality of care in patients with multiple clinical conditions: summary of a conference conducted by the Society of General Internal Medicine. J Gen Intern Med. 2007;22(8): 1206-11.

8. Heath I, Rubinstein A, Stange KC, van Driel ML. Quality in primary health care: a multidimensional approach to complexity. BMJ. 2009;338:b1242.

9. Smith SM, Soubhi H, Fortin M, Hudon C, O'Dowd T. Managing patients with multimorbidity: systematic review of interventions in primary care and community settings. BMJ. 2012;345:e5205.

10. Miller WL, Crabtree BF, Nutting PA, Stange KC, Jaen CR. Primary care practice development: a relationship-centered approach. Ann Fam Med. 2010;8(Suppl 1):S68-79; s92.

11. Dattalo M, Nothelle S, Tackett S, et al. Frontline account: targeting hot spotters in an internal medicine residency clinic. J Gen Intern Med. 2014;29(9):1305-7.

12. Koroukian SM, Warner DF, Owusu C, Given CW. Multimorbidity redefined: prospective health outcomes and the cumulative effect of cooccurring conditions. Prev Chronic Dis. 2015;12:E55.

13. Bayliss EA, Bonds DE, Boyd CM, et al. Understanding the context of health for persons with multiple chronic conditions: moving from what is the matter to what matters. Ann Fam Med. 2014;12(3):260-9.

14. Uijen AA, van de Lisdonk EH. Multimorbidity in primary care: prevalence and trend over the last 20 years. Eur J Gen Pract. 2008;14(Supp 1):28-32.

15. Starfield B, Shi L, Macinko J. Contribution of primary care to health systems and health. Milbank Q. 2005;83(3):457-502.

16. Stange KC, Etz RS, Gullett $\mathbf{H}$, et al. Metrics for assessing improvements in primary health care. Annu Rev Public Health. 2014;35:423-42.

17. Scott JG, Cohen D, Dicicco-Bloom B, Miller WL, Stange KC, Crabtree BF. Understanding healing relationships in primary care. Ann Fam Med. 2008;6(4):315-22. 